\title{
Gas characterisation based on a snapshot interferometric imaging spectrometer
}

\author{
Aneline Dolet ${ }^{\mathrm{a}, \mathrm{b}}$, Daniele Picone ${ }^{\mathrm{a}}$, Mauro Dalla Mura ${ }^{\mathrm{a}, \mathrm{c}}$, Didier Voisin ${ }^{\mathrm{b}}$, Silvère Gousset ${ }^{\mathrm{d}}$, \\ Sylvain Doutéd ${ }^{\mathrm{d}}$, and Etienne Le Coarer ${ }^{\mathrm{d}}$ \\ ${ }^{a}$ GIPSA-lab, Univ. Grenoble Alpes, CNRS, Grenoble INP, GIPSA-lab, Grenoble, France \\ ${ }^{b} U G A$, CNRS, Institut des Géosciences de l'Environnement (IGE), Grenoble, France \\ 'Tokyo Tech World Research Hub Initiative (WRHI), School of Computing, Tokyo Institute of \\ Technology, Tokyo, Japan \\ ${ }^{\mathrm{d} I n s t i t u t ~ d e ~ P l a n e ́ t o l o g i e ~ e t ~ d ' A s t r o p h y s i q u e ~ d e ~ G r e n o b l e ~(I P A G), ~ G r e n o b l e, ~ F r a n c e ~}$
}

\begin{abstract}
Modern atmospheric gas monitoring applications demand progressively better performances with regards to spatial, spectral and temporal resolutions. In this context, great potential is shown by a newly developed family of cutting-edge snapshot imaging spectrometers based on Fabry-Perot interferometry, whose conceptual design was patented under the name ImSPOC. Three sensor prototypes based on the ImSPOC concept are under development addressing different spectral ranges and applications: 1) one in the near infrared wavelength range for $\mathrm{CH}_{4}$ or $\mathrm{H}_{2} \mathrm{~S}$ detection, 2) one in the ultra-violet and visible range for $\mathrm{NO}_{2}, \mathrm{O}_{4}, \mathrm{O}_{3}$ and $\mathrm{O}_{2}$ characterisation and 3) one specifically for $\mathrm{CO}_{2}$ monitoring. After the realisation of these prototypes there is the need arose to provide intelligible and well-calibrated acquisitions for the final users. This study presents the ImSPOC concept from the signal processing point of view, framing the optical transformations performed in the instruments under an appropriate mathematical model formulation. Additionally, preliminary developments are presented to address the first step of the signal processing pipeline for this instrument: the estimation of the thickness of each interferometer. This is a fundamental step for obtaining calibrated acquisitions that could then be used for gas monitoring.
\end{abstract}

Keywords: Fourier-Transform Imaging Spectroscopy, Hyperspectral imaging, Sensor calibration, Gas monitoring, Spectroscopy

\section{INTRODUCTION}

In recent decades, remote sensing has become central for monitoring the Earth's surface (its lands, urbanisation, oceans, agriculture, etc.) and its atmosphere ${ }^{1-4}$ In particular, the need for accurate measurements of gases in the atmosphere is ever increasing for tasks as monitoring climate change and air quality study and regulation issues. These tasks require data acquisitions with ever higher spatial, spectral and temporal resolutions, motivating the development of new sensors and their corresponding signal processing methods.

Hyperspectral imaging is a technique usually used for applications as atmospheric gas monitoring. ${ }^{4}$ Today, different types of hyperspectral imaging systems, based on different techniques (e.g., spatial, snapshot or spectral scanning $^{5}$ ), are available and are tailored to specific applications. In particular, monitoring atmospheric gases in the context of climate change requires increasingly accurate sensors and less spaced acquisitions. Most current hyperspectral imaging systems are based on a trade-off of price and spectral, spatial and temporal resolution. Such a compromise must then be overcome by new technologies. The miniaturisation of sensors is also increasingly targeted for their use airborne or equipping nanosatellites. ${ }^{6,7}$ Then, it allows costs reduction: production costs as well as their costs of use, e.g, with a less expensive launch for a nanosatellite than for a conventional satellite.

This study focuses on a groundbreaking hyperspectral image acquisition technology based on a miniaturised static (snapshot) interferometric imaging spectrometer, called Imaging SPectrometer On Chip (ImSPOC). ${ }^{8}$ It is

Contact e-mail: aneline.dolet@grenoble-inp.fr 
composed of several Fabry-Perot interferometers of different thicknesses, distributed in a matrix and superimposed on an imaging system that is composed of an array of detectors placed in the focal plane of a series of micro-lenses. A Fabry-Perot interferometer is composed of two parallel slabs where the light is refracted inside or transmitted depending on the considered ray, allowing the acquisition of the same light but with different optical paths inside the interferometer. The difference of distances covered by two different light rays is called optical path difference (OPD). The detectors then acquire a set of images, which can be interpreted as replicas of the same scene, each having a multiplicative modulation induced by the interferometric fringes, whose frequency depends on the thickness of each interferometer. The different OPDs, associated with each interferometer, provide a set of interferometer samples for each pixel of the imaged scene, which can be inverted to reconstruct a hyperspectral image. This design allows a competitive spectral resolution at a relatively low cost compared to conventional remote sensing systems for measuring gases with similar performance.

Despite the high performance of the ImSPOC sensor, there are many challenges in processing the acquired data to obtain gas measurements. First, the calibration of the joint design of this set of interferometers is challenging. Each non ideal element of the instrument presents some variability in their characteristics that introduce spatially incoherent behaviours between the different acquired images. For example, the parallax effect caused by the specific angle of view of the scene of each interferometer or small differences in the manufacture of each micro-lens or interferometer. In addition, theoretically ideal interferograms cannot be obtained in practice due to manufacturing and physical constraints, e.g, in theory, the different OPDs have thicknesses that increase linearly to allow the acquisition of linearly sampled interferograms, which is not the case in reality.

The role of signal processing in obtaining an accurate estimate of the spectrum per pixel from the acquired interferograms is therefore of utmost importance. Ad-hoc methods must be developed for sensor calibration as well as for spectrum reconstruction, in case the measured interferogram is not directly usable for end-user applications. As a final step, the reconstructed spectrum must be processed to provide an accurate measurement of the concentration of the gas of interest.

As the ImSPOC system is still under development, only a few prototypes in two different wavelength ranges are available at the moment: ultraviolet/visible (UV/VIS) and near infrared (NIR). ${ }^{8}$ In this context and concerning the image processing part, only the calibration of the ImSPOC sensor has been started and a part of it is presented here. Therefore, in this paper we address the measurements of the interferometer thicknesses. First of all, the ImSPOC system is detailed as well as its mathematical formulation. The method and experiments performed to estimate the thicknesses of the interferometers of one prototype as well as the achieved results are then presented before concluding on this study. The future studies necessary for the continuation of the developments are finally discussed.

\section{SENSOR DESCRIPTION}

\subsection{The ImSPOC prototype}

Fabrication details depend on the wavelength range aimed at in a given prototype. The following description applies to the prototype used for this particular study which operates in the UV/VIS wavelengths. The considered ImSPOC $^{8}$ prototype is composed of: a leading optical system (Figure 1(a)) to restrict the incident wavelength range, a matrix of Fabry-Perot interferometers with different thicknesses (Figure 1(b)), a matrix of micro-lenses (Figure 1(c)) and a photodetector matrix placed at the micro-lenses focal plane (Figure 1(d)).

The ImSPOC instrument was developed to be used on nanosatellite fleets. So, it is as small as possible (see Figure 2) while maintaining a correct alignment between all components. Each photodetector gives then an image of the scene multiplied by a modulation made of interferometric fringes whose frequency depends on the OPD, and therefore on the thickness, of each interferometer (Figure 3). These modulated images are called thumbnails to keep the word image for the sensor data output which is the collection of all thumbnails. A specific part of the scene, corresponding to a particular pixel (for example, the pixel highlighted in red in the Figure 3(left)), therefore gives a reflectance for each thumbnail that is also modulated by the interferometric fringes. This can be seen in the Figure 3(centre) where the red pixel mentioned above is surrounded in green in some thumbnails. It allows to reconstruct an interferogram for each pixel of the scene. 

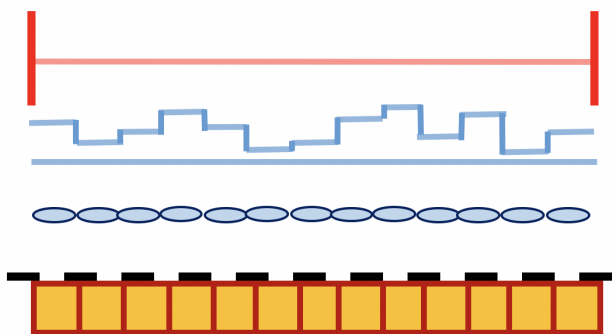

(a)

Figure 1. Scheme of the ImSPOC prototype used in this study with all its components: (a) the leading optical system, (b) the Fabry-Perot interferometer matrix, (c) the micro-lens matrix and (d) the photodetector matrix which is on the focal plane (in black dashes). The scheme does not give any indication about the proportion between the different components.
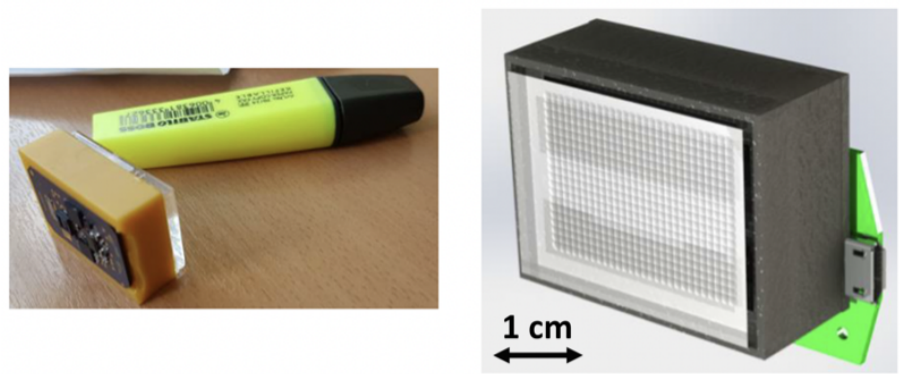

Figure 2. Picture (left) and model (right) of an ImSPOC instrument.

\subsection{Acquisition model}

\subsubsection{Wave theory}

The optical rays that will pass through the ImSPOC system can be modelled with the framework of plane waves interacting with layered media. ${ }^{9}$ In particular, it is customary to represent a certain monochromatic plane wave propagating, at a fixed frequency $\nu$, with the following complex wavefunction:

$$
U(\mathbf{r})=A \exp (-j \mathbf{k} \cdot \mathbf{r}),
$$

where the spatial position $\mathbf{r}$ is the direction whose modulus $r$ is the distance from a certain observer, $A$ is the complex envelope, which is a complex value $A=|A| \exp (j \arg \{\mathbf{A}\})$ and $\mathbf{k}$ is the wavevector, whose modulus $k$ is called angular wavenumber and whose unit vector $\mathbf{k} / k$ is the direction of propagation.

In plane waves, the wavefronts, that are the surfaces with constant phase, are parallel planes perpendicular to $\mathbf{k}$. The distance $\lambda$ between two consecutive surfaces with the same phase is fixed and is called wavelength:

$$
\lambda=\frac{2 \pi}{k}=\frac{c}{\nu} .
$$

For planar waves, the frequency $\nu$ is constant for every material, but the phase velocity $c=c_{0} / n$ varies $\left(c_{0}\right.$ is the speed of light in the void and $n$ the refraction index of the material of propagation), hence also $\lambda$ and $k$.

The optical intensity is then defined as: ${ }^{9}$

$$
I(\mathbf{r})=|U(\mathbf{r})|^{2}=U(\mathbf{r}) U^{\star}(\mathbf{r}) .
$$

where $U^{\star}$ is the complex conjugate of $U$. 


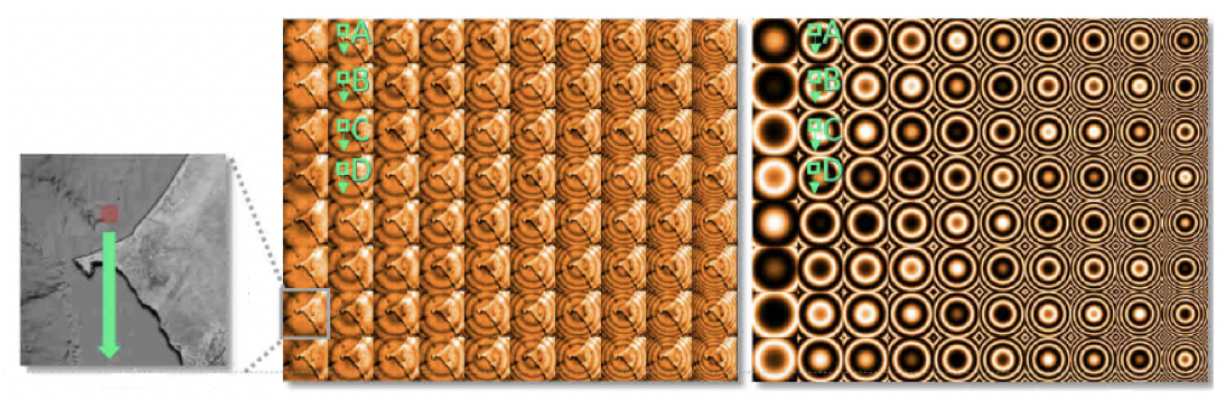

Figure 3. Left: The imaged scene with a particular pixel highlighted in red. Center : The image acquired by the sensor with all the thumbnails that are replicas of the scene multiplied by the interferometric fringes corresponding to each interferometer. The pixel of interest is surrounded by a green line in some thumbnails. Right: Image of only the modulation of the interferometric fringes for each interferometer. The same pixel is also surrounded by a green line in some thumbnails.

\subsubsection{Fourier Transform Spectroscopy}

As the ImSPOC system is composed of a matrix of interferometers, the interferometry theory is recalled here. First, consider only two monochromatic waves with complex amplitudes $U_{1}(\mathbf{r})=\sqrt{I_{1}} \exp \left(j \phi_{1}\right)$ and $U_{2}(\mathbf{r})=$ $\sqrt{I_{2}} \exp \left(j \phi_{2}\right)$, with their respective intensities $I_{1}=\left|U_{1}\right|^{2}$ and $I_{2}=\left|U_{2}\right|^{2}$. The phase difference $\phi=\phi_{2}-\phi_{1}$ can be rewritten in terms of the time delay $\tau$ of propagation of the two waves in the interferometer, $\phi=2 \pi \nu \tau$. It is also common to rewrite the aforementioned time delay in terms of the so-called OPD, which is defined as $\delta:=\tau c_{0}$ (:= is used to denote "equal by definition"). A physical interpretation of the OPD is given in section 2.2.3. The intensity $I$ of the combined wave $U=U_{1}+U_{2}$, applying Equation 3, allows to obtain the following interference intensity:

$$
\begin{aligned}
I & =\left|U_{1}+U_{2}\right|^{2}=\left|U_{1}\right|^{2}+\left|U_{2}\right|^{2}+U_{1}^{\star} U_{2}+U_{1} U_{2}^{\star} \\
& =I_{1}+I_{2}+2 \sqrt{I_{1} I_{2}} \cos \phi,
\end{aligned}
$$

If we suppose that the second wave is also subject to a certain amplitude attenuation factor $0 \leq R<1$, we can express the intensity of the first and second beam respectively as $I_{1}=I_{0}$ and $I_{2}=R^{2} I_{0}$. As the phase difference is $\phi=k \delta=2 \pi \sigma \delta$ (see Equation 2), the combined wave has the following intensity (coming from Equation 4):

$$
\begin{aligned}
I & =\left(1+R^{2}+2 R \cos \phi\right) I_{0} \\
& =\left(1+R^{2}+2 R \cos (2 \pi \sigma \delta)\right) I_{0} .
\end{aligned}
$$

In real life applications, incoming waves generated by the same source are characterised by different wavelengths which exhibits uncorrelated fluctuations. To combine these sources, a quasi-monochromatic source has to be assumed, ${ }^{10,11}$ meaning that the operating range of wavelengths is small compared to the central wavelength. With this assumption, the previous discussion can be extended by integrating over the range of wavenumbers $\left[\sigma_{\min }, \sigma_{\max }\right]$. Taking as example the model of two waves interferometry, the final contribution, by integrating Equation $5 \mathrm{~b}$ over $\sigma=\nu / c$, is:

$$
I(\delta)=\int_{\sigma_{\min }}^{\sigma_{\max }}\left(1+R^{2}(\sigma)\right) I_{0}(\sigma) d \sigma+2 \int_{\sigma_{\min }}^{\sigma_{\max }} R(\sigma) I_{0}(\sigma) \cos (2 \pi \sigma \delta) d \sigma .
$$

The diagram $I(\delta)$, obtained by varying the values of the OPD, is known as interferogram. A common way to tackle this inversion problem under the Fourier transform framework is to make the following assumptions:

- the support of the incident waves is completely limited to the range of wavenumbersf $\left[\sigma_{\min }, \sigma_{\max }\right]$, which allows to extend the integration bounds to $[0,+\infty]$;

- the expression of $R(\sigma)=R$ has no dependency with regards to the wavenumber (or wavelength). 
Under these assumptions, the first term of the right hand side of Equation 6 can be rewritten in terms of $I(\delta)$ for $\delta=0$, specifically $I(0)=(1+R)^{2} \int_{0}^{+\infty} I_{0}(\sigma) d \sigma$. We can then reorder the terms of Equation 6 to highlight the variable part of the output $I^{\prime}(\delta)$ :

$$
\begin{aligned}
I^{\prime}(\delta): & =\frac{1}{2 R}\left(I(\delta)-\frac{1+R^{2}}{(1+R)^{2}} I(0)\right) \\
& =\int_{0}^{+\infty} I_{0}(\sigma) \cos (2 \pi \sigma \delta) d \sigma .
\end{aligned}
$$

Equation 7b can be seen as a Fourier Cosine Transform from the spectrum $I_{0}(\sigma)$ (in the domain of wavenumbers) to a linear transformation $I^{\prime}(\delta)$ of the interferogram $I(\delta)$ (in the domain of the OPDs).

Inverse Fourier transform of Equation $7 \mathrm{~b}$ gives: ${ }^{12}$

$$
I_{0}(\sigma)=4 \int_{0}^{+\infty} I^{\prime}(\delta) \cos (2 \pi \sigma \delta) d \delta
$$

which allows to reconstruct the spectrum. As Fourier transform is involved, this procedure is commonly known as Fourier Transform Spectroscopy (FTS). ${ }^{12}$

\subsubsection{Optical path difference in Fabry-Perot interferometers}

Fabry-Perot interferometry allows to generate an interferometry pattern by introducing an OPD between consecutive emerging ray beams, due to multiple reflection inside an optical system. ${ }^{13}$ One of the most common configurations, also employed in the ImSPOC prototype, consists in an optical cavity bounded by two parallel reflecting surfaces. Two consecutive reflections within the surfaces of the cavity introduce an attenuation factor and a time delay in the propagation of the transmitted waves.

As a physical interpretation, we can see the OPD as the product of the geometric length of the difference between the paths followed by light beams through a given system and the index of refraction of the medium through which it propagates. ${ }^{13}$ Visually, a simple model to identify the OPD in Fabry-Perot interferometer is shown in Figure 4: $\theta_{0}$ is the transmission angle of the ray emerging from the interferometer, $\theta$ is the refraction angle within the interferometer, $L$ is the thickness of the interferometer, $n$ is the refraction index within the interferometer and $n_{0}$ is the refraction index outside the interferometer.

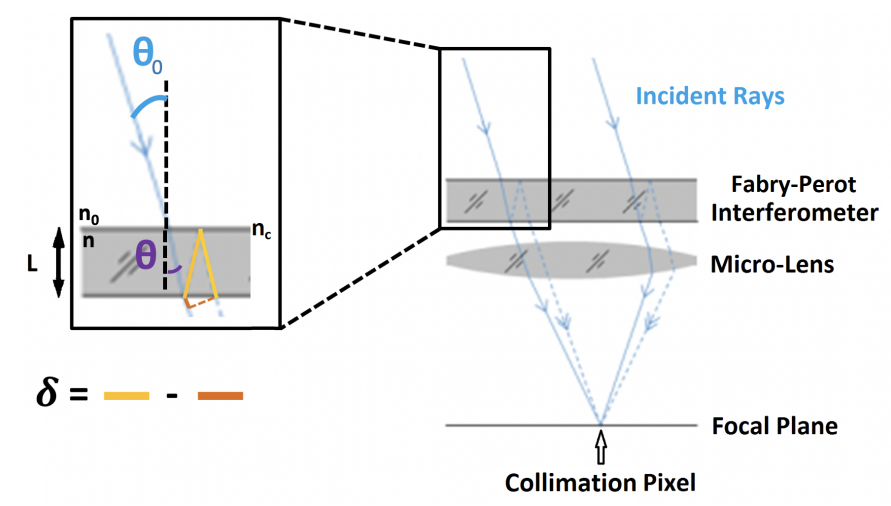

Figure 4. Structure of a parallel faces interferometer, showing its OPD $\delta$.

The OPD may be obtained by analysing the geometrical paths starting from the spot in which the rays split. Focusing on two consecutive emerging rays, the first ray (orange path in Figure 4 ) traces a distance $2 L \tan \theta \sin \theta_{0}$, while the second (yellow path in Figure 4) $2 L / \cos \theta$ :

$$
\delta=n \frac{2 L}{\cos \theta}-n_{0}\left(2 L \tan \theta \sin \theta_{0}\right) \approx n\left(\frac{2 L}{\cos \theta}-2 L \tan \theta \sin \theta\right)=2 n L \cos \theta,
$$


where the approximation is valid either for small $\theta_{0}$ or if $n_{0} \approx n$. The latter is verified in most practical design as Fabry-Perot, which are usually composed by glass slabs (whose reflection index is very similar to the air's) with a surface coating treatment that increases their reflectance. This also automatically implies that $\theta_{0} \approx \theta$ according to Snell's law. Additionally, the attenuation factor $R$ defined in section 2.2 .2 can be seen in the interferometer as the reflectance in the sense of Fresnel of the internal side surface of the cavity: ${ }^{9}$

$$
R=\left|\frac{n-n_{c}}{n+n_{c}}\right|^{2}
$$

where $n_{c}$ is the reflectivity index of the coating material. For the ImSPOC prototype, glass slabs are treated with reflective thin film of $\mathrm{TiO}_{2} \cdot{ }^{8}$

\subsubsection{ImSPOC model}

The ImSPOC concept is described by a matrix of $N$ low-finesse Fabry-Perot interferometers with different thickness stacked over an imaging system, which is composed by a matrix of lenses and a focal plane. In its conception, the instrument can be seen as $N$ spatial replicas of the configuration shown in Figure 4, disposed next to each other in a 2D-array. Each interferometer features a different thickness, allowing the device to register various samples of the interferogram with a single snapshot acquisition.

The incident ray $I_{i n}\left(\theta_{i j}, \sigma\right)$ can be characterised in the spectral and spatial domains. The spectral dependency is expressed as a function of the wavenumber $\sigma$. In the spatial domain, as a function its incidence angle $\theta_{i j}$ that is defined as the solid angle range of the rays that, after collimation, excites the photodetector on the focal plane in the position $(i, j)$. The collimation system, shown on the right of Figure 4 is ideally the same for all sub-images, hence we can assume that the $(i, j)$ position is the same for each thumbnail; this condition has to be verified during the calibration phase to avoid misalignment. A leading optical system (Figure 1(a)) allows to limit the range of incident wave within the desired wavenumber range $\left[\sigma_{\min }, \sigma_{\max }\right]$ and within a given solid angle to minimise the effect of cross-talk among different interferometers.

Each element of the instrument can be modeled with its own transfer function, yet the characterization of the leading and collimating optical system, of the quantum efficiency of the sensors and their point spread function are outside the scope of this paper and we will assume them to be transparent for the system.

We will focus instead on the transfer function of the Fabry-Perot interferometer; in particular the intensity of the acquisition $I_{\text {out }}\left(\delta_{b}, i, j\right)$ registered by the detector with coordinates $(i, j)$ associated to the $b$-th thumbnail can be modelled as:

$$
I_{\text {out }}\left(\delta_{b}, i, j\right)=\int_{\sigma_{\min }}^{\sigma_{\max }} I_{i n}\left(\theta_{i j}, \sigma\right) T_{F P, b}\left(\theta_{i j}, \sigma\right) d \sigma
$$

where:

- $R(\sigma)$ is the reflectivity of the surface of the interferometer;

- $T_{F P, b}\left(\theta_{i j}, \sigma\right)=T_{F P D, b}\left(\theta_{i j}, \sigma\right)\left(1+R^{2}(\sigma)+2 R(\sigma) \cos \left(2 \pi \sigma \delta_{b}\right)\right)$ is the transmission function of the Fabry-Perot interferometer; its expression comes from Equation 5 with an added term $T_{F P D, b}\left(\theta_{i j}, \sigma\right)$ to take care of the transfer function from the incident light to the first directly transmitted beam ( $I_{0}$ in the original equation);

- $\delta_{b}=2 n L_{b} \cos \left(\theta_{i j}\right)$ is the OPD between the directly transmitted ray and the one transmitted after a double reflection in the $b$-th interferometer (see Equation 9).

- $L_{b}$ is the thickness of the $b$-th interferometer

By fixing a certain pixel position $(i, j)$, Equation 11 can be inverted with the technique and assumptions described in section 2.2.2 to recover the spectrum $I_{i n}\left(\theta_{i j}, \sigma\right)$. Unfortunately given the limited amount of available thumbnails, the available version $I_{\text {out }}\left(\delta_{b}, \theta_{i j}\right)$ of the interferogram is sampled at discrete values $\left\{\delta_{b}\right\}_{b=1, \ldots N}$ of the OPD and the reconstructed spectrum may also be obtained as a sampled version via Fast Fourier Transform. To be able to correctly place the samples on the interferogram, it is hence mandatory to accurately estimate the real thickness $L_{b}$ of each interferometer, as $\delta_{b}$ is a function of it. 


\section{INTERFEROMETER THICKNESS ESTIMATION}

The thickness estimation for each interferometer of the considered ImSPOC prototype is presented here. It is the first calibration step that has to be done before any other data processing. Indeed, because of the non ideal precision of the fabrication, the thicknesses of the interferometers could differ from the theoretical ones. However, knowing the real thicknesses is of major importance to be sure to reconstruct coherently sampled interferograms.

\subsection{Theory}

In this context we are characterizing each Fabry-Perot cavity separately and the input sources are a series of monochromatic incident ray beams with fixed incidence angle, each characterized by a different central wavelenght, whose range is limited by the characteristics of the employed monochromator, i.e. [250,950] nm. For this purpose, opposed to section 2.2.2, it is more convenient to characterize the resulting interferogram $I_{b}(\sigma)$ in terms of the wavenumber $\sigma$ instead of the OPD, which is theoretically fixed for each element of the matrix. Its expression, under the assumptions made in section 2.2 .2 , can be obtained by recasting eq. $5 \mathrm{~b}$ as:

$$
I_{b}(\sigma)=I_{0}\left(1+R^{2}+2 R \cos \left(2 \pi \sigma \delta_{b}\right)\right)
$$

where the value of $\delta_{b}$, assumed fixed for each interferometer, is equal, according to eq. 9, to:

$$
\delta_{b}=2 n L_{b} \cos \theta \approx 2 L_{b},
$$

where last step is obtained supposing the incident ray is perpendicular to the interferometer surface, which means $\theta=0$, and because the refraction index within the cavity is approximately equal to its value in the void $(n \approx 1)$.

The target of the calibration is to estimate the value of $L_{b}$, hence $\delta_{b}$ is unknown. A quick check of the expression of eq. 12 shows that it is periodic with period $\delta_{b}$ in the domain of the wavelenghts $\lambda=1 / \sigma$.

Real acquisitions will feature some non idealities compared to the simplified formula we consider here, those include non-uniformity of the surfaces of the Fabry-Perot, spectral aberration due to variations of $R$, cross-talk among different cavities, noise effects in the photodectors. Even if $I_{b}(\sigma)$ is not strictly periodic, some techniques to highlight periodic behaviours can be used to provide an estimation $\hat{\lambda}_{b}$ of $\delta_{b}$. One of those techniques is the so called Spectral Density Estimation.

The procedure is as follows:

- Input: Interferogram $I_{b}(\sigma)$, composed by a certain number of samples in the interval $\left[\sigma_{\min }, \sigma_{\max }\right]$, incidence angle $\theta$ of the monochromator.

- Calculate the Discrete Fourier Transform $F_{b}(\lambda)=\sum_{\sigma=\sigma_{\min }}^{\sigma_{\max }} I_{b}(\sigma) \exp (2 \pi \sigma \lambda)$

- Identify the peak value $\hat{\lambda}_{b}=\arg \max _{\lambda>\lambda_{\min }}\left|F_{b}(\lambda)\right|$, where $\lambda_{\min }$ is appropriately chosen to avoid the region around the low wavelenghts, which are characterized by highest correlation

- Estimate the thickness of the $b$-th interferometer, according to eq. 13 , as $\hat{L}_{b}=\frac{\hat{\lambda}_{b}}{2 n \cos \theta}$

As $\left|F_{b}(\lambda)\right|^{2}$ represents the PSD of the interferogram, this method is equivalent to find the information of the periodicity in the original domain as embedded in the autocorrelation coefficient of the acquired signal.

\subsection{Experiments}

Thickness calibration will be performed in our study with a specific setup; to allow spectral diversity, the interferometers have to be illuminated by a quasi-monochromatic light source. In particular, a halogen lamp (with an almost black body behaviour) was coupled with a mechanical monochromator to produce a quasimonochromatic light beam with tunable wavelength. This quasi-monochromatic light is collimated in an optical fiber and divided in two identical light beam by a cube beamsplitter. One of the light beam shines on the ImSPOC instrument, carefully aligned to assume a direction of incidence of the light ray on the instrument as normal as possible. The second light beam is acquired by a grating spectrometer (USB2000+, Ocean Optics), which operates from $340 \mathrm{~nm}$ to $1030 \mathrm{~nm}$ with a $1 \mathrm{~nm}$ spectral resolution. The spectrometer allows more precise 
measurement than the system embedded in the mechanical monochromator and is employed in this setup to acquire the reference central wavelength of the generated light and to serve as a wavelength reference for our ImSPOC acquisition. The acquisition scheme is shown Figure 5.

In our experiments, the wavenumbers' acquisitions range is from $10000 \mathrm{~cm}^{-1}$ to $20000 \mathrm{~cm}^{-1}$ (or equivalently $[500 ; 1000] \mathrm{nm}$ for wavelengths), with $100 \mathrm{~cm}^{-1}$ steps.

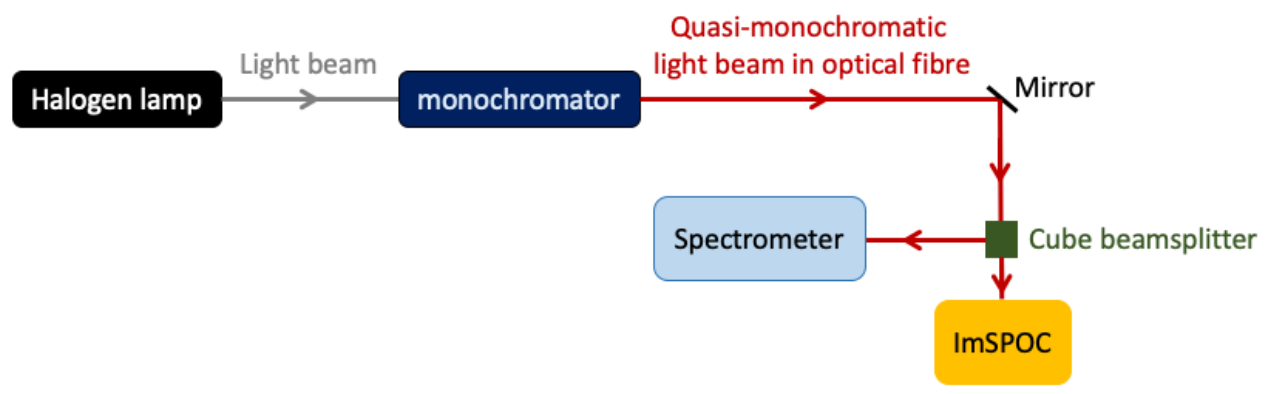

Figure 5. Acquisition scheme: the halogen lamp is coupled with a mechanical monochromator to produce a quasimonochromatic light beam which is redirected both in a grating spectrometer and towards the ImSPOC instrument using a cube beamsplitter.

\subsection{Method}

The first step of the data processing is to extract the central wavelengths of each acquisition from the spectrometer; to line up with the framework proposed in section 3.1, we will take the reciprocal of these values to express them in wavenumbers $\sigma$.

Subsequently, for each interferometer (the $b$-th one), we will identify the associated thumbnail (the $b$-th thumbnail) over the acquired image; the mean value over the thumbnail will be calculated for each acquired wavelength. The evolution of the averaged amplitude over wavenumbers, called $I_{b}(\sigma)$, represents the acquired $b$-th interferogram.

$I_{b}(\sigma)$ will be composed of 101 samples, which are extended with zero-padding up to 601. This operation allows a finer scale when performing the Fourier transform and obtain $F_{b}(\lambda)$. As explained in section 3.1, the estimated OPD corresponds to the value of $\lambda$ for which $\left|F_{b}(\lambda)\right|$ is maximum, ignoring the fundamental peak.

\subsection{Validation}

The thicknesses of the interferometers have been manufactured approximately in the range $[2 ; 48] \mu \mathrm{m}$ with a linear increase.

The test that has been performed is to verify the degree of linearity of the vector of estimated thicknesses ordered according to their nominal increasing order suggested by the manufacturer: $\hat{\mathbf{L}}=\left\{\hat{L}_{1}, \ldots, \hat{L}_{N}\right\}$.

This linearity can be quantified with the root mean square error (RMSE) of its linear regression. Specifically we define linear regression of $\mathbf{L}$ the linear function $\mathbf{R}=\left\{R_{1}, \ldots, R_{N}\right\}$, obtained by fitting the above with the least squared approach, in other words by minimizing the following RMSE:

$$
R M S E=\|\hat{\mathbf{L}}-\mathbf{R}\|_{2}=\sqrt{\sum_{b=1}^{N}\left(\hat{L}_{b}-R_{b}\right)^{2}} .
$$




\section{RESULTS}

The type of images acquired at each wavelength is shown Figure 6 where the optical fiber beam can be seen for each thumbnail. For a particular wavelength (e.g. in the previously cited Figure: 586 nm), all interferometer are not highlighted exactly with the same energy (the thumbnails at the extremity have much lower intensity for each wavelength). As some thumbnails' amplitudes are too low to be taken in consideration, just a subset of the available elements are considered in this study (within the white limits in Figure 6). Each snapshot is normalized in the range $[0 ; 1]$ to equalize the energy balance along different wavelengths.

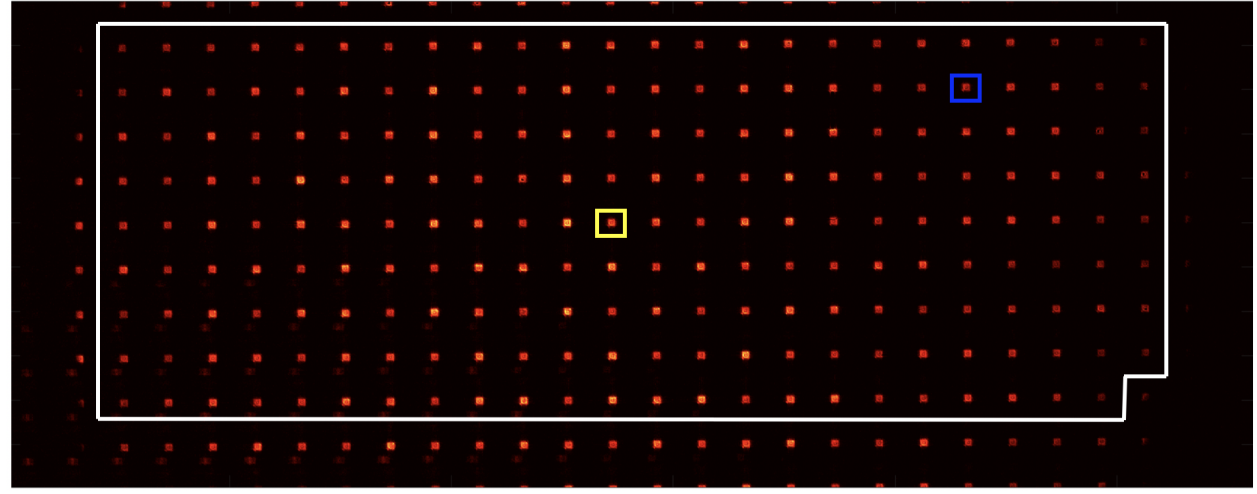

Figure 6. Acquired image at $586 \mathrm{~nm}$ after the amplitude normalisation. The thumbnails considered in this study are within the white borders.

For all the 215 thumbnails that are studied here, their amplitudes over the wavenumbers $I_{b}(\sigma)$ are extracted. Figure 7 shows the $I_{b}(\sigma)$ evolution for the thumbnail surrounded by blue rectangle on Figure 6 and its Fourier transforms $\left|F_{b}(\lambda)\right|$. The estimated OPD $\hat{\lambda}_{b}$ is then the position of the maximal value excluding the fundamental peak, framed in red in fig. 7 .
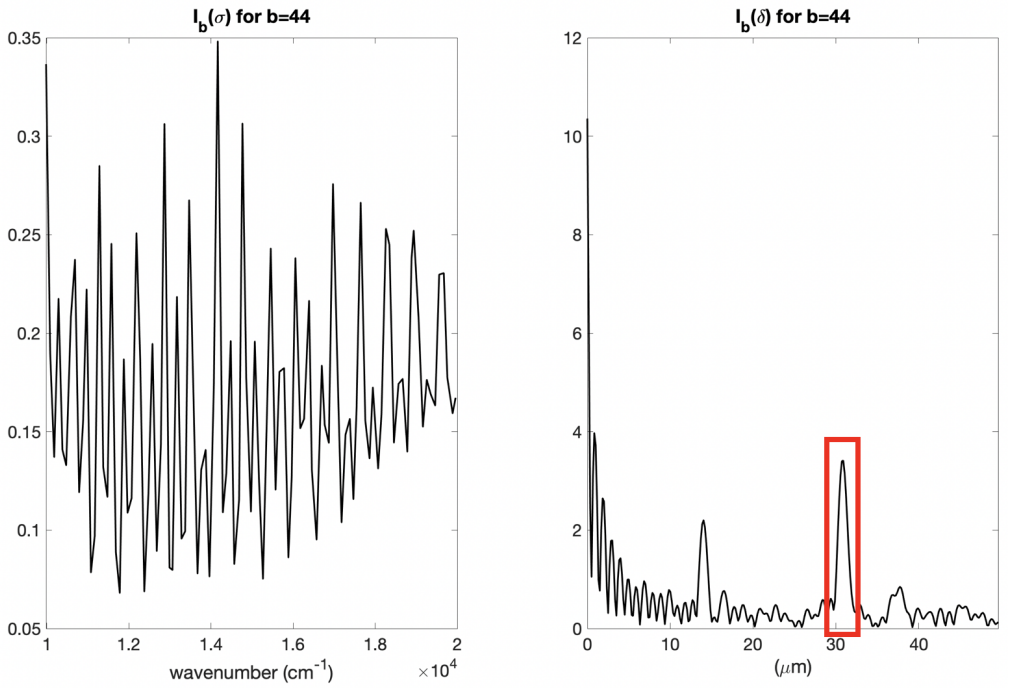

Figure 7. Left: $I_{b}(\sigma)$ evolution for the thumbnail surrounded by blue rectangle on Figure 6. Right: the corresponding Fourier transforms $I_{b}(\lambda)$. The estimated OPD is the position of the peak surrounded in red.

The same procedure is repeated for all the thumbnails. However, for the lowest thicknesses of the interferometers (thumbnail line of the thumbnail surrounded with yellow rectangle in Figure 6 ), the $I_{b}(\sigma)$ evolution on the acquisition range $[10000 ; 20000] \mathrm{cm}^{-1}$ does not return a signal that can be treated as an approximately periodical signal. 
The main issue, an example of which is shown in Figure 8 and corresponding to the yellow framed thumbnail on Figure 6), is that the resolution of $100 \mathrm{~cm}^{-1}$ used for the interferogram is too coarse, which doesn't allow to distinguish between the width of the principal lobe and the peak identifying the period.
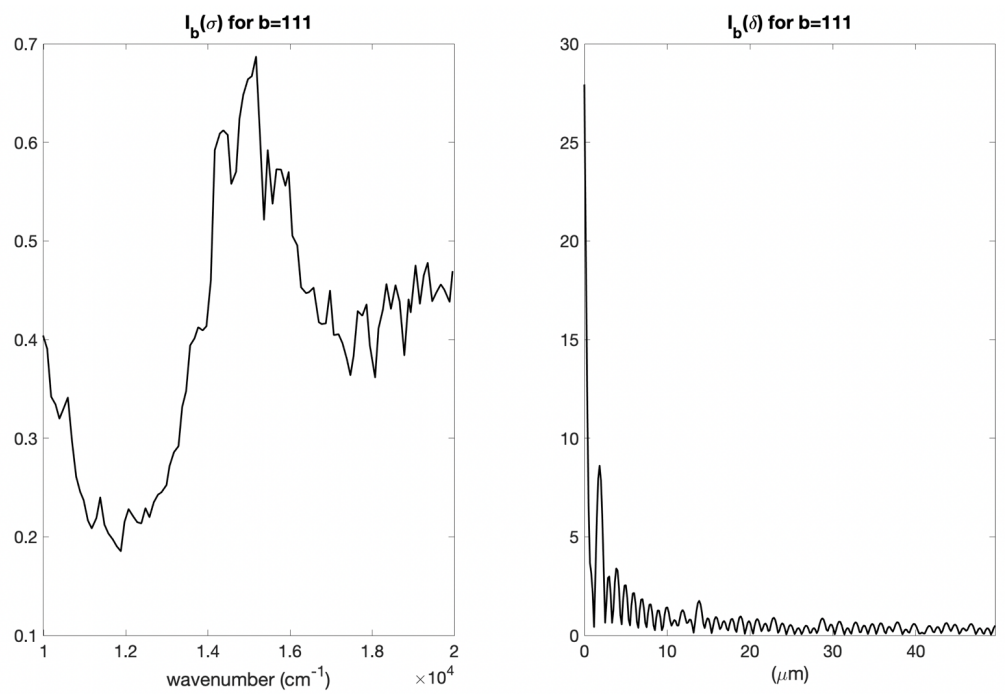

Figure 8. Left: $I_{b}(\sigma)$ evolution for the thumbnail surrounded by yellow rectangle on Figure 6 . Right: the corresponding Fourier transforms $\left|F_{b}(\lambda)\right|$.

The estimated OPD for all the thumbnails are shown Figure 9 and are in the range $[1.5 ; 46.9] \mu \mathrm{m}$. As mentioned in section 3.4, the theoretical OPDs should be in the range $[2 ; 48] \mu \mathrm{m}$ which means that the method probably somewhat underestimates the OPDs. Also, the increase of the OPDs can be plotted and the resulting plot should be linear (see Figure 10). The linear regression is plotted in red in this Figure and the error between the estimated OPDs and this linear regression is of $R M S E=4.14$, calculated with Equation 14. This Figure highlights then a good linearity but also that more than one thumbnails, thus interferometers, have the same OPDs which is impossible in reality. Figure 11 shows for each estimated OPD the number of thumbnails that have been estimated with this OPD.

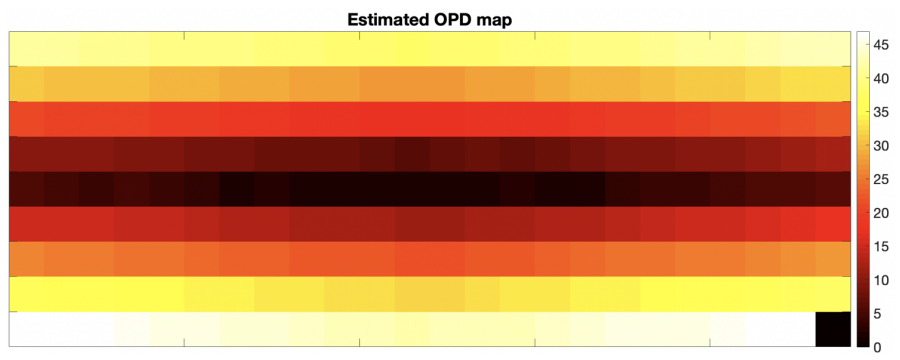

Figure 9. Estimated OPDs map in the range $[1.5 ; 46.9] \mu \mathrm{m}$.

Finally, the validation of the method can be done looking at the order of the thumbnails, which means the order of the increasing OPDs of the interferometers. Both estimated OPD and theoretical order maps are presented Figure 12. This Figure highlights the similarity of these both maps. However, a map of the differences is shown Figure 13. There, the label for each thumbnail corresponds to the differences between the orders of the estimated and the theoretical OPD values. The highest differences are at both upper corners, which are parts from the images where the amount of light is lowest (see Figure 6), which is then completely understandable. Anyway, the maximum error value is of 6 under 215 thumbnails which is an encouraging result. Indeed, these errors can come from really small differences in terms of OPDs values. 


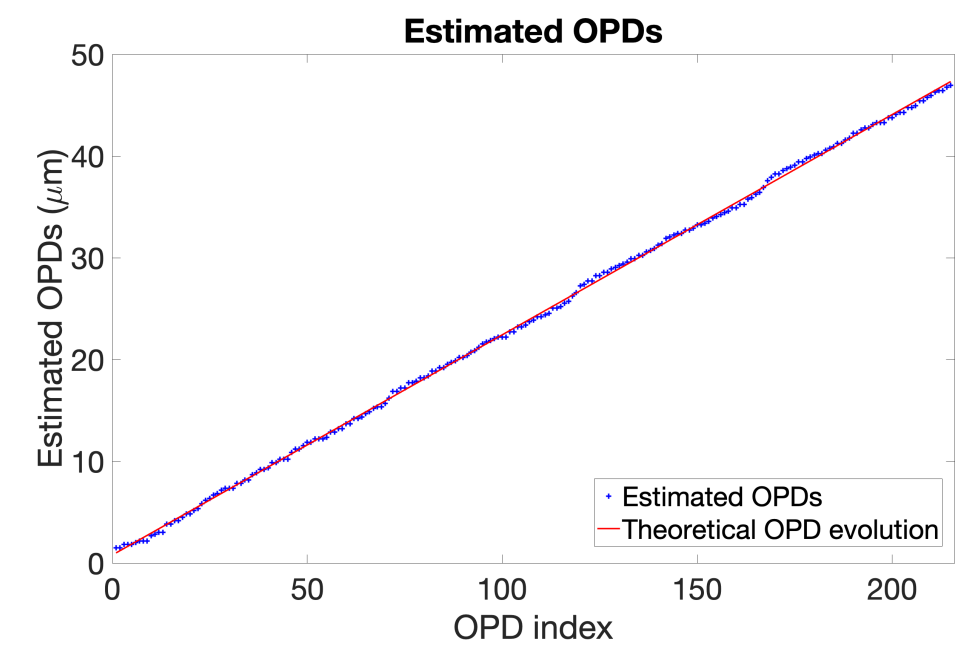

Figure 10. Estimated OPDs plot over the thumbnail indexes. The red curve is the linear regression of the estimated OPDs.

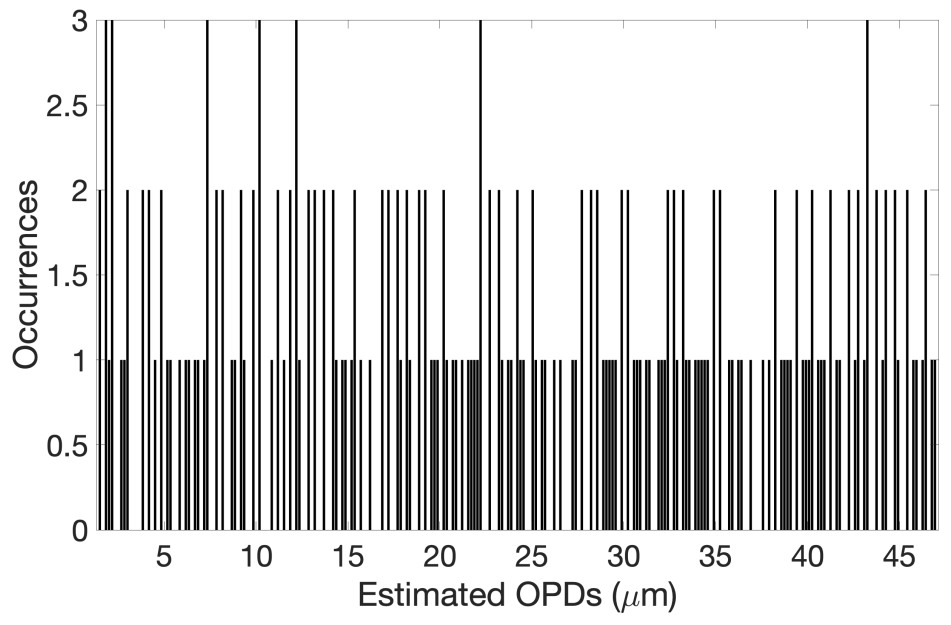

Figure 11. Occurrences of each estimated OPD.

\section{CONCLUSION AND DISCUSSION}

This study describes how to estimate the interferometer thicknesses of a ImSPOC sensor. The preliminary results of this method are encouraging as they led to measure quasi-linearly increasing thicknesses over the interferometers. This is a vital concept for the proper function of the device under test.

In addition, the order of the thumbnails is overall estimated with relatively good accuracy. The main issue at the moment is to improve the resolution in the estimation of the thickness. This would also have the beneficial effect to avoid to have the same value associated to multiple interferometers, which is not possible due to the design of the instrument.

In future works, this estimation should be improved by performing some ad hoc experiments with a controlled uniform illumination over the entire field of view of the instrument. Also, acquiring images on a wider range of wavenumbers and with smaller step sizes will allow to improve the results, particularly for the smallest thicknesses. Nevertheless, this first experiment already allows a good estimation of the largest thicknesses. However, to validate the estimation, it is of major importance to compare the estimated values to the nominal ones which is currently under development. 


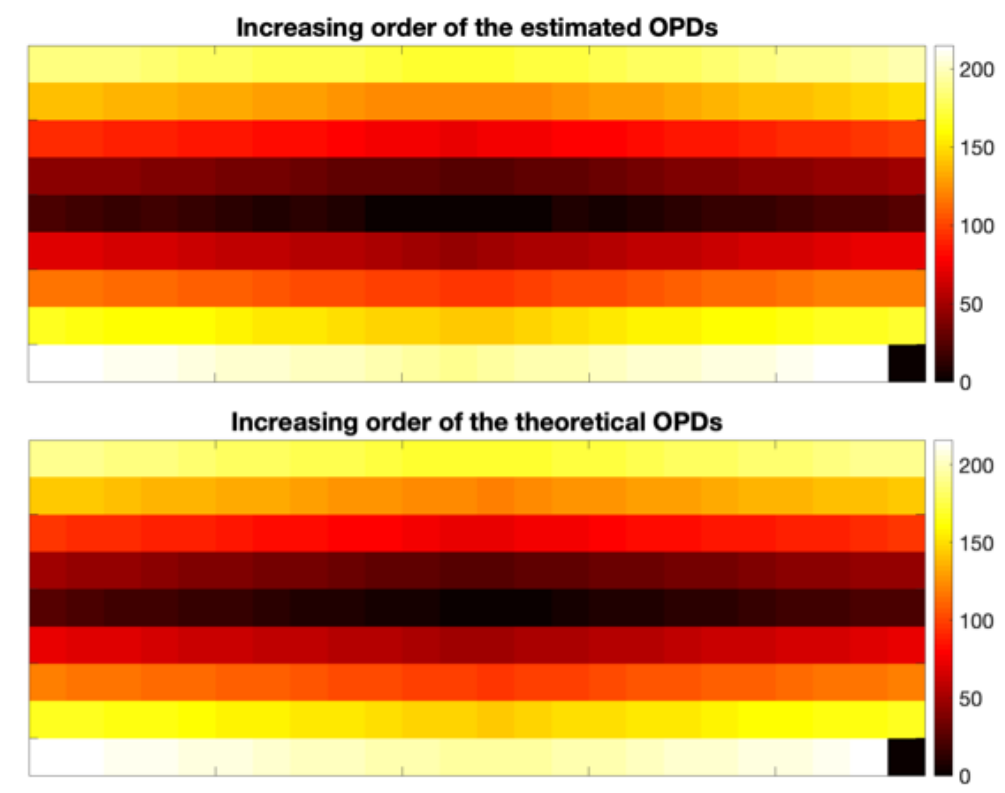

Figure 12. These maps show the order of the thumbnails in an increasing OPD, then thicknesses, of the interferometers sens. The up one is based on the estimated OPD where the bottom one come from the theoretical OPDs.

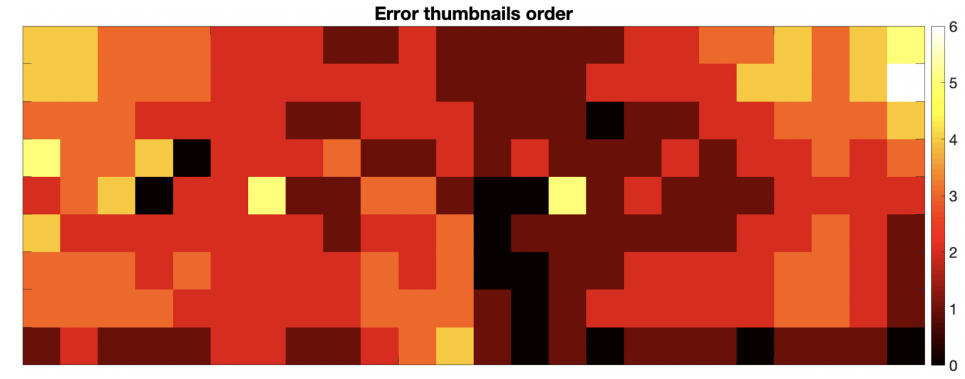

Figure 13. This map shows the differences, in terms of OPDs order, between the theoretical and the estimated orders.

Further pre-processing needs to be developed for overcoming the spatial distortion present in each thumbnail caused by non ideal optical elements and alignment. This is very important to reconstruct a coherent thumbnail 3D dataset and then, with the accurate thicknesses estimation, coherent interferogram for each pixel of the acquired scene. Another critical processing is to accurately reconstruct a spectrum from an interferometer for each pixel. This implies to have a specific model to inverse properly the interferograms. To this end, the model of section 2.2.4 will be tested. All these steps will be of utmost importance in order to acquire calibrated data that could then be exploited for gas characterisation.

\section{ACKNOWLEDGMENTS}

This work was supported by funds from the AuRA region and FEDER under the project ImSPOC-UV and IMAGAZ and by the European Union's H2020 research and innovation program under grant agreement SCARBO No 769032 .

\section{REFERENCES}

[1] Tilling, A. K., O'Leary, G. J., Ferwerda, J. G., Jones, S. D., Fitzgerald, G. J., Rodriguez, D., and Belford, R., "Remote sensing of nitrogen and water stress in wheat," (October 1, 2007). 
[2] Ben-Dor, E., Chabrillat, S., Dematt, J. A. M., Taylor, G. R., Hill, J., Whiting, M. L., and Sommer, S., "Using imaging spectroscopy to study soil properties," Remote Sensing of Environment, Imaging Spectroscopy Special Issue 113, S3855 (2009).

[3] Adam, E., Mutanga, O., and Rugege, D., "Multispectral and hyperspectral remote sensing for identification and mapping of wetland vegetation: A review," Wetlands Ecology and Management 18, 281-296 (06 2010 ).

[4] Kim, Y. C., Yu, H.-G., Lee, J.-H., Park, D.-J., and Nam, H.-W., "Hazardous gas detection for ftir-based hyperspectral imaging system using dnn and cnn," In Electro-Optical and Infrared Systems: Technology and Applications XIV, International Society for Optics and Photonics , 10433:1043317 (2017).

[5] Bouy, C., Robin, T., and d'Humires, B., "Spectral imaging end-users needs, market and trends," tech. rep., Tematys, Exploration of photonics markets (January 2018).

[6] Pola Fossi, A., Miniaturization of an infrared hyperspectral camera, theses, Université Paris-Saclay (Dec. 2016).

[7] Shay, A., August, I. Y., and Stern, A., "Compressive and classical hyperspectral systems: a fundamental comparison," in [Compressive Sensing IV], Ahmad, F., ed., SPIE (may 2015).

[8] Guerineau, N., Le Coarer, E., Ferrec, Y., and De La Barriere, F., "Spectro-imageur multivoie transforme de fourier," (Jan. 2018). FR patent 1,656,162.

[9] Saleh, B. E. A. and Teich, M. C., [Fundamentals of Photonics], John Wiley \& Sons, Inc. (aug 1991).

[10] Thorne, A., Litzén, U., and Johansson, S., [Spectrophysics: principles and applications], Springer Science \& Business Media (1999).

[11] Hariharan, P., [Optical Interferometry, 2e], Elsevier (2003).

[12] Hariharan, P., [Basics of interferometry], Elsevier (2010).

[13] Hecht, E., [Optics (5th Edition)], Pearson (2016). 